Journal Club

Editor's Note: These short, critical reviews of recent papers in the Journal, written exclusively by graduate students or postdoctoral fellows, are intended to summarize the important findings of the paper and provide additional insight and commentary. For more information on the format and purpose of the Journal Club, please see http://www.jneurosci.org/misc/ifa_features.shtml.

\title{
The Role of Prefrontal Mixed Selectivity in Cognitive Control
}

\author{
Araceli Ramirez-Cardenas and Pooja Viswanathan \\ Animal Physiology, Institute of Neurobiology, University of Tübingen, Auf der Morgenstelle 28, 72076 Tübingen, Germany and International Max-Planck \\ Research School for Cognitive and Systems Neuroscience, 72074 Tübingen, Germany \\ Review of Blackman et al.
}

Cognitive control is the ability to modify responses to a given stimulus according to changing contexts or goals (Miller and Cohen, 2001). It encompasses diverse functions, including working memory, attention, context and goal processing, and response inhibition. Given the multifaceted nature of cognitive control, addressing its neurophysiological basis in a structured protocol can be challenging. However, such study is necessary to understand how we solve problems in real life, where even simple tasks require many cognitive functions simultaneously, while we integrate information "on the fly" according to current goals and needs.

How various cognitive control mechanisms are implemented and coordinated in the brain is a long-standing question. Studies in human and nonhuman primates point to the prefrontal cortex (PFC) as a key area in building cognitive control (MacDonald et al., 2000; Miller, 2000; Koechlin et al., 2003). But how do prefrontal neurons implement the required cognitive flexibility? Mixed neuronal selectivity has been proposed as an efficient way to expand the coding capabilities of neuronal populations (Stokes, 2011;

\footnotetext{
Received June 6, 2016; revised July 19, 2016; accepted July 21, 2016

We thank Andreas Nieder and Felix Moll for comments on the manuscript.

The authors declare no competing financial interests.

Correspondence should be addressed to either Araceli Ramirez-

Cardenas or Pooja Viswanathan, Department of Animal Physiology, Institute of Neurobiology, University of Tübingen, Auf der Morgenstelle 28, 72076, Tübingen, Germany. E-mail: araceli.ramirezcardenas@student.uni-tuebingen.de or pooja.vis@gmail.com.

DOI:10.1523/JNEUROSCI.1816-16.2016

Copyright $\odot 2016$ the authors $\quad 0270-6474 / 16 / 369013-03 \$ 15.00 / 0$
}

Mante et al., 2013; Rigotti et al., 2013; Fusi et al., 2016). Neurons that respond to diverse combinations of task variables (including stimulus features, rules, and decisions) have been identified in the PFC (Warden and Miller, 2010; Stokes, 2011; Mante et al., 2013; Rigotti et al., 2013). The response patterns of these multitasking neurons change over the course of a trial and could enable cognitive flexibility.

In a paper published in The Journal of Neuroscience, Blackman et al. (2016) recorded prefrontal single-cell activity while monkeys performed a task that demands cognitive control. The authors used a modified version of the AX continuousperformance task applied in clinical settings. In the original task, different letters are flashed in a continuous stream. Subjects are instructed to respond whenever the letter X follows an A. In the dot pattern expectancy task (Blackman et al., 2016, their Fig. $1 A, C$ ), letter stimuli are substituted with dot patterns (Jones et al., 2010) and the continuous nature of the task is replaced by a trial structure. On each trial, monkeys were shown a cue stimulus, followed by a delay and then a probe stimulus. The cue stimulus could either be the specific target dot pattern A or one of several nontarget patterns collectively called B. Similarly, the probe stimulus could either be the specific target pattern X or one of the dot patterns referred as Y. Monkeys were required to respond with a left movement to the target pattern combination (A-cue, X-probe) and with a right movement for any other combination of cue and probe dot patterns (AY, BX, and
BY trials). The cue type (A or one of B) provided the context in which the identity of the following probe was either relevant or irrelevant for the response. In A-cue trials, the identity of the probe pattern is needed to select a response. In B-cue trials, the response can already be determined in the cue period as any following probe (X or $\mathrm{Y}$ ) would require a rightward response.

The task was administered in two kinds of trial blocks. In balanced blocks, the four types of trials (AX, AY, BX, and BY) were presented with the same frequency. In prepotent blocks, target trials (AX) were overrepresented to increase the subjects' tendency to respond impulsively to nontarget combinations. In A-cue trials, response inhibition was required when a nontarget $\mathrm{Y}$ dot pattern was presented in the probe period. In B-cue trials, response inhibition is demanded in trials involving the target $\mathrm{X}$ dot pattern.

Three results of this study make important contributions to the current discussion on the neuronal dynamics in PFC. The first concerns the encoding of the cue stimulus in the delay period. The task requires the cue identity to be held in working memory and integrated with information concerning the subsequent probe. The classical view of working memory holds that task-relevant stimulus information is maintained online by persistent activity of prefrontal neurons. According to this view, one would expect a considerable proportion of neurons with sustained selective activity during the delay period, after the cue disappears. However, the authors found only some of these classi- 
cal "delay neurons" (Blackman et al., 2016, their Fig. 3A,E). Moreover, at the population level, a decoding approach (Blackman et al., 2016, their Fig. $7 A, B$ ) showed that cue information gradually decayed during the delay period. These findings challenge the classical view regarding the implementation of working memory and suggest that additional mechanisms maintain stimulus information (Lebedev et al., 2004; Stokes et al., 2013; Jacob and Nieder, 2014; Lara and Wallis, 2014).

Second, instead of delay neurons, Blackman et al. (2016) found many cueselective neurons that responded either in the cue or in the probe period. In B-cue trials, activity in these neurons increased when the cue was presented, whereas in A-cue trials, activity increased in the probe period. This pattern was reflected by a change in the neurons' preferred cue along the course of a trial. Interestingly, most cue-selective neurons preferred the B-cue in the cue period. This bias disappeared in the delay period and reversed to A during the probe presentation. The neurons that exhibited this particular pattern of activity were called switch neurons. The authors argue that this response pattern indicates that switch neurons are most active when cognitive control is demanded. In A-cue trials, probe identity ( $\mathrm{X}$ vs $\mathrm{Y}$ ) is crucial to determine the correct response, whereas in B-cue trials, cue discrimination is comparatively more important. Notably, the switching pattern was stronger in prepotent blocks than in balanced blocks, further suggesting a relation with cognitive control (Blackman et al., 2016, their Fig. 9).

Switch neurons exhibit the time-specific selectivity frequently observed in multitasking neurons. Moreover, the pattern of their responses can be characterized as selective to diverse task variables (i.e., cue and probe type) in different trial periods. However, mixed selectivity at the single-neuron level increases the representational capacity in the population only when neuronal response patterns are diverse. So, even though the response of switch neurons is illustrative of such diversity, coding characteristics can be better evaluated in whole populations (Miller and Fusi, 2013; Stokes, 2015). Furthermore, there is evidence that PFC neurons cannot be grouped into distinct classes according to their temporal response profiles. Instead, temporal profiles in a population lie in a continuum (Miller and Fusi, 2013; Rigotti et al., 2013). In light of these findings, one should keep in mind the limited scope of conclusions obtained from a functionally defined subpopulation
(Raposo et al., 2014) like the switch neurons. Therefore, the authors present a view of the whole population of recorded neurons by applying a demixed principal component analysis (Blackman et al., 2016, their Fig. $8 B)$. Most of the variance in the identified components could be attributed to time or the interaction between time and trial type (cue-probe sequence). This shows that the population strongly encodes trial time. Moreover, it suggests that different cueprobe combinations are encoded differently in the different phases of the trial.

The third main contribution that Blackman et al. (2016) make is the report of a context-dependent representation of the probe stimulus. Particularly, whether the probe was encoded seemed to depend on the identity of the previous cue. The identity of the probe was relevant for a successful outcome only in A-cue trials. The authors trained a classifier on the firing activity of probe-selective neurons (Blackman et al., 2016, their Fig. 6) and found that prefrontal population activity better discriminated probe type (X vs $\mathrm{Y}$ ) in A-cue trials than in B-cue trials. This result illustrates how selectivity can represent a changing context at the population level.

Overall, the authors suggest that stimuli and conditions that demand more cognitive control are overrepresented in prefrontal neurons (or specific subpopulations). AY trials, when a prepotent response must be inhibited, provide evidence for the link between neuronal activity and monkeys' behavior. In these trials, switch neurons responded more strongly and monkeys showed longer reaction times. The behavioral relevance of prefrontal switch neurons was not, however, directly tested. It is probable that neurons with other response patterns also contribute to behavior. Comparing neuronal activity or selectivity in correct versus error trials could offer stronger evidence for the role of prefrontal neuronal activity in cognitive control.

It would also be interesting to analyze the extent to which the variance of visual stimuli affected neuronal responses. In contrast to the A-cue and the X-probe, which were specific dot patterns, both the B-cue and Y-probe comprised different patterns collectively designated as nontarget $\mathrm{B}$ and $\mathrm{Y}$ (Blackman et al., 2016, their Fig. 1C). These stimuli (B-cues and Y-probes) also elicited stronger neuronal responses in the cue and probe period, respectively. Thus, it is quite possible that the neurons responded to the greater variance in these stimulus sets over the single dot patterns A and X and only indirectly to the cognitive control engaged in these trials. Therefore, closer examination of neuronal activity in response to the individual $\mathrm{B}$ and $\mathrm{Y}$ dot patterns would help dissociate the effects of stimulus variance and cognitive control.

In conclusion, the present study offers clues about how cognitive flexibility is built in PFC. The results show that multitasking prefrontal neurons flexibly represent task variables at the population level. In light of growing evidence for the role of mixed selectivity in an adaptive and dynamic population code (for review, see Fusi et al., 2016), this study opens exciting future avenues. How are task-relevant and -irrelevant features differentiated in this dynamic encoding? How is this all-inclusive representation read out to select the appropriate behavioral response? Could classically tuned cells play a role in this translation? How does PFC interact with other high-order and lower-level areas? The authors may be able to address this last question with data collected simultaneously from the posterior parietal cortex using this protocol. Finally, beyond its valuable contribution to our understanding of cognitive control, the study shows the merits of applying a task used in clinical settings to an animal model. Such translations allow interventions that would otherwise be impossible in humans (Blackman et al., 2013) and provide great insight into normal and impaired cognition.

\section{References}

Blackman RK, Macdonald AW 3rd, Chafee MV (2013) Effects of ketamine on contextprocessing performance in monkeys: a new animal model of cognitive deficits in schizophrenia. Neuropsychopharmacology 38: 2090-2100. CrossRef Medline

Blackman RK, Crowe DA, DeNicola AL, Sakellaridi S, MacDonald AW 3rd, Chafee MV (2016) Monkey prefrontal neurons reflect logical operations for cognitive control in a variant of the AX continuous performance task (AX-CPT). J Neurosci 36:4067-4079. CrossRef Medline

Fusi S, Miller EK, Rigotti M (2016) Why neurons mix: high dimensionality for higher cognition. Curr Opin Neurobiol 37:66-74. CrossRef Medline

Jacob SN, Nieder A (2014) Complementary roles for primate frontal and parietal cortex in guarding working memory from distractor stimuli. Neuron 83:226-237. CrossRef Medline

Jones JA, Sponheim SR, MacDonald AW 3rd (2010) The dot pattern expectancy task: reliability and replication of deficits in schizophrenia. Psychol Assess 22:131-141. CrossRef Medline

Koechlin E, Ody C, Kouneiher F (2003) The architecture of cognitive control in the human prefrontal cortex. Science 302:1181-1185. CrossRef Medline 
Lara AH, Wallis JD (2014) Executive control processes underlying multi-item working memory. Nat Neurosci 17:876-883. CrossRef Medline

Lebedev MA, Messinger A, Kralik JD, Wise SP (2004) Representation of attended versus remembered locations in prefrontal cortex. PLoS Biol 2:e365. CrossRef Medline

MacDonald AW 3rd, Cohen JD, Stenger VA, Carter CS (2000) Dissociating the role of the dorsolateral prefrontal and anterior cingulate cortex in cognitive control. Science 288:18351838. CrossRef Medline

Mante V, Sussillo D, Shenoy KV, Newsome WT (2013) Context-dependent computation by recurrent dynamics in prefrontal cortex. Nature 503:78-84. CrossRef Medline
Miller EK (2000) The prefontral cortex and cognitive control. Nat Rev Neurosci 1:59-65. CrossRef Medline

Miller EK, Cohen JD (2001) An integrative theory of prefrontal cortex function. Annu Rev Neurosci 24:167-202. CrossRef Medline

Miller EK, Fusi S (2013) Limber neurons for a nimble mind. Neuron 78:211-213. CrossRef Medline

Raposo D, Kaufman MT, Churchland AK (2014) A category-free neural population supports evolving demands during decision-making. Nat Neurosci 17:1784-1792. CrossRef Medline

Rigotti M, Barak O, Warden MR, Wang XJ, Daw ND, Miller EK, Fusi S (2013) The importance of mixed selectivity in complex cogni- tive tasks. Nature 497:585-590. CrossRef Medline

Stokes M (2011) The spatiotemporal structure of population coding in monkey parietal cortex. J Neurosci 31:1167-1169. CrossRef Medline

Stokes MG (2015) "Activity-silent" working memory in prefrontal cortex: a dynamic coding framework. Trends Cogn Sci 19: 394-405. CrossRef Medline

Stokes MG, Kusunoki M, Sigala N, Nili H, Gaffan D, Duncan J (2013) Dynamic coding for cognitive control in prefrontal cortex. Neuron 78:364-375. CrossRef Medline

Warden MR, Miller EK (2010) Task-dependent changes in short-term memory in the prefrontal cortex. J Neurosci 30:1580115810. CrossRef Medline 